\title{
Study on Magnetic Memory Testing Signal Characteristics of Pipeline Defects Based on Wavelet Packet Analysis
}

\author{
Shujun Liu ${ }^{1} \mathrm{a}^{*}$, Shenglin $\mathrm{Li}^{1}$, Ming Jiang ${ }^{1}$, Dean $\mathrm{He}^{1}$ \\ ${ }^{1}$ Logistics Engineering University, Chongqing 401311, China \\ a119042856@qq.com
}

\begin{abstract}
Keywords: Magnetic memory testing, Signal characteristics, Stress concentration, Defect, Wavelet packet energy spectrum.
\end{abstract}

\begin{abstract}
At present, the judging principle of Magnetic Memory Testing technology (MMT) can only indicate the stress concentration zone, but can not get information from the stress concentration further. In the paper, to get information from the stress concentration zone, a MMT signal analysis method based on wavelet packet energy spectrum is proposed. The specimen tension load experiment shows that when the tension load is $200 \mathrm{MPa}$, the signal wavelet packet energy spectrum distributes uniformly, the percentage of separate energy to total energy is lower than $15 \%$. When the tension load is $410 \mathrm{MPa}$, the maximum wavelet packet energy locate in 1, 3 and 4 spectrum, the percentage of 1 4 energy to total energy is $73.8 \%$, and the main wavelet packet energy locates in the low frequency area. After the specimen yields, the maximum wavelet packet energy locates in 1 and 2 spectrum; the percentage of $1 \sim 3$ energy to total energy is $87.3 \%$. The distribution of wavelet packet energy spectrum is very separate, and the main wavelet packet energy locates in 1 and 2 spectrum. If the stress concentration degree is low, then the wavelet packet energy spectrum distribution is equal; if the stress concentration degree is high, then the wavelet packet energy spectrum distribution is concentrated, and the main energy is concentrated in low frequency area.
\end{abstract}

\section{Introduction}

Ferromagnetic metal materials have been widely used in industry, and the material damage is also getting more and more attention. As an important means of energy transmission, Petroleum and natural gas pipeline transportation's safe operation is also highly valued. Traditional nondestructive testing methods, such as ultrasound, magnetic flux leakage, X-ray, etc, can find the macro or most obvious defects which have been developed such as Crack, fold and inclusion, etc, by testing the key parts of the material, equipment and components. However, it is difficult to realize effective prediction for the early damage of equipment and components in service. In 1997, Russian scholar Doubov first proposed the metal magnetic memory testing technology. The principle can be expressed as: For the effect of the load, the domain organization orientation will cause magnetostriction properties and irreversible reorientation in the Ferromagnetic components working in a geomagnetic field. The maximum leakage magnetic field is formed in the stress and deformation zone. The tangential component of the magnetic field in the stress concentration area $H_{P}(x)$ has the maximum value, while the sign of the normal component $H_{P}(y)$ changes and has zero-crossing position. The irreversible change of the magnetic state remains after the elimination of the working load. By the testing of the magnetic flux leakage field's normal component $H_{P}(y)$ and the Calculation of gradient, the stress concentration position of the component can be exactly deduced [1,2]. A large number of engineering practice $[3,4]$ shows that the existing criterion by the characteristic of the normal component of the magnetic field and its gradient may lead to the omission of the detection or error detection. In addition, just indicating the stress concentration position is far from enough for pipeline maintenance. Thus, it is necessary to obtain more abundant information from magnetic memory 
signals for the damage assessment and the remaining life prediction of pipelines, such as the degree of stress concentration, the geometric parameters of the defect, etc.

\section{Wavelet Packet Energy Spectrum}

Wavelet is a new method developed in the last ten or twenty years [5]. Because of its good time-frequency localization, it can overcome the shortcomings of Fourier transform and get a wide range of applications. Wavelet packet analysis is a better method than wavelet analysis. The frequency band is divided into several layers, and the high frequency part of the wavelet is further decomposed, So as to improve the time-frequency resolution. Wavelet packet analysis is to project the signal into a set of orthogonal wavelet function of the sub space, reaching the target of the expansion of the signal at different scales and extracting the characteristics of the signal in different frequency bands. Each band contains a wealth of information. Its energy distribution can be expressed by different characteristics of the signal. To analyze the characteristics of each frequency band energy, and then to extract the effective feature vector, various types of stress concentration or defect can be identified.

Assuming that the wavelet packet decomposition of the signal $S$ is $\mathrm{j}$ layers, the signal is decomposed into $j$ layers and $2^{j}$ nodes [6]. Supposing that the wavelet packet decomposition coefficient series for a certain frequency band is $\left\{x_{k}\right\}, k=1,2 \cdots n$, the wavelet packet energy of this band is $E_{j m}=\sum_{k=1}^{n}\left(x_{k}\right)^{2} m=0,1 \ldots 2^{j}-1$. Forming a feature vector $\left[E_{j 0}, E_{j 1}, \cdots, E_{j 2^{j}-1}\right]$ with the energy elements in each bands of the $j$ layer, the characteristic vector $\left[E_{j 0}, E_{j 1}, \cdots, E_{j 2^{j}-1}\right]$ is called as the wavelet packet energy spectrum of the signal, record as $\left[E_{j m}\right]$. The sum of the energy of the signal in each frequency band is equal to that of the original signal. The magnetic memory signals in each frequency band characterize the characteristic information of the original signal in the frequency range. Various factors such as the type of pipe defects or the distribution of stress concentration have great influence on the energy of each frequency band. When the energy is large, and then $\left[E_{j m}\right]$ is usually a relatively large value. Thus, it may cause some inconvenience in data analysis. To compare the energy distribution of magnetic memory signals with different types of defects, the wavelet packet energy spectrum needs to be normalized. The normalized wavelet packet energy spectrum $\left[E_{j m} / E\right]$ of the signal $S$ can be obtained after the Normalization of $\left[E_{j m}\right]$. The total energy of the signal $S$ is written as $E=\sqrt{\sum_{m=0}^{2^{j}-1} E_{j m}}$.

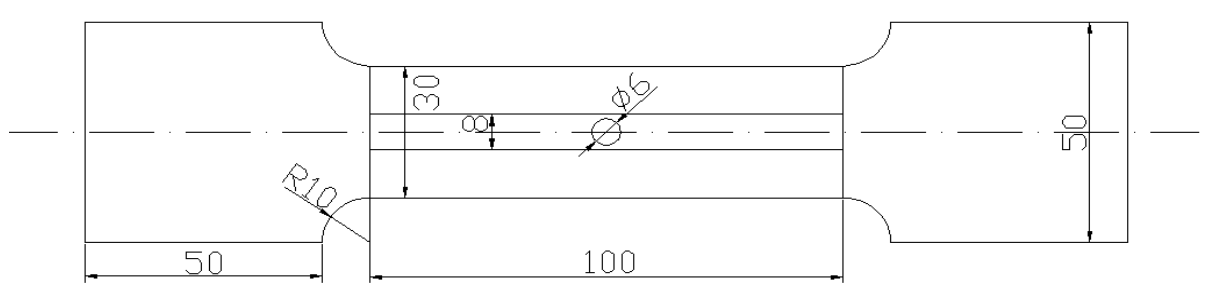

Fig. 1 Schematic of specimen and detecting lines.

\section{Experiment}

Specimen. The experimental material is taken from X60 grade pipeline steel. According to GB6397-1986 metal tensile test specimen, the material is processed into a sheet shaped tensile specimen as shown in Fig 1. The Specimen thickness is $4 \mathrm{~mm}$, and a circular hole with a diameter of $6 \mathrm{~mm}$ is processed in the center of the specimen, in order to generate local stress concentration in the 
process of drawing. Taking into account the residual stress in the process of leveling, the specimen should be annealed at first after being processed, in order to avoid the initial state of the magnetic memory signal interference.

As shown in Fig. 1, two horizontal lines on the surface of the test piece are drawn, the distance from which to the center of the circular hole is $4 \mathrm{~mm}$. The vertical line represents the starting point and end point measurement at both ends.

Experimental instrument. The experiment was carried out on the MTS810 type hydraulic servo testing machine. In the experiment the test piece is perpendicular to the ground, and Chuck speed is controlled to $1 \mathrm{~mm} / \mathrm{min}$. The magnetic memory signal on the surface of the specimen was collected by the MFL-4032 magnetic flux leakage / magnetic memory detector [7], which was developed by the Institute of logistics engineering and Eddysun Electronics Co., Ltd. The non magnetic clamping device clamping double channel magnetic memory testing probe is driven by the motor to collect the measuring line along the measuring line in fixed moving speed and lift off height $0.5 \mathrm{~mm}$. In the course of the movement, the probe is perpendicular to the surface of the specimen. In order to avoid environmental interference, during the experiment, all the magnetic memory tests were carried out on the aluminum alloy in the same direction on the same position. The test platform is placed in an open position in the laboratory, Away from all kinds of components and devices that can excite the magnetic field, so as not to be interfered by external magnetic field.

Test results and analysis. The magnetic memory signals were collected by $200 \mathrm{MPa}, 410 \mathrm{MPa}$ and 420MPa, respectively. The signal curve after denoising [8] is shown in Fig. 2.

3 layer wavelet packet decomposition of the signal with DB2 wavelet is carried out, and $2^{3}$ orthogonal wavelet spaces are generated. The signal is divided into 8 sub bands that do not overlap. The 1 4 band is the low frequency band of the signal, and 5 8 band is the high frequency band of the signal. $f / \mathrm{Hz}$ is the signal sampling frequency, and the frequency range of each frequency band is shown in Table 1.

Table 1 . The bands corresponding to frequency range.

\begin{tabular}{cccccccc}
\hline $\mathrm{S} / \mathrm{N}$ & frequency $/ \mathrm{Hz}$ & $\mathrm{S} / \mathrm{N}$ & frequency $/ \mathrm{Hz}$ & $\mathrm{S} / \mathrm{N}$ & frequency $/ \mathrm{Hz}$ & $\mathrm{S} / \mathrm{N}$ & frequency $/ \mathrm{Hz}$ \\
\hline 1 & $0 \sim f / 16$ & 2 & $f / 16 \sim f / 8$ & 3 & $f / 8 \sim 3 f / 16$ & 4 & $3 f / 16 \sim f / 4$ \\
5 & $f / 4 \sim 5 f / 16$ & 6 & $5 f / 16 \sim 3 f / 8$ & 7 & $3 f / 8 \sim 7 f / 16$ & 8 & $7 f / 16 \sim f / 2$ \\
\hline
\end{tabular}

The normalized wavelet packet energy spectrum of the magnetic memory signal after 3 layers of wavelet packet decomposition is expressed in the form of histogram, as shown in Fig. 3-5.

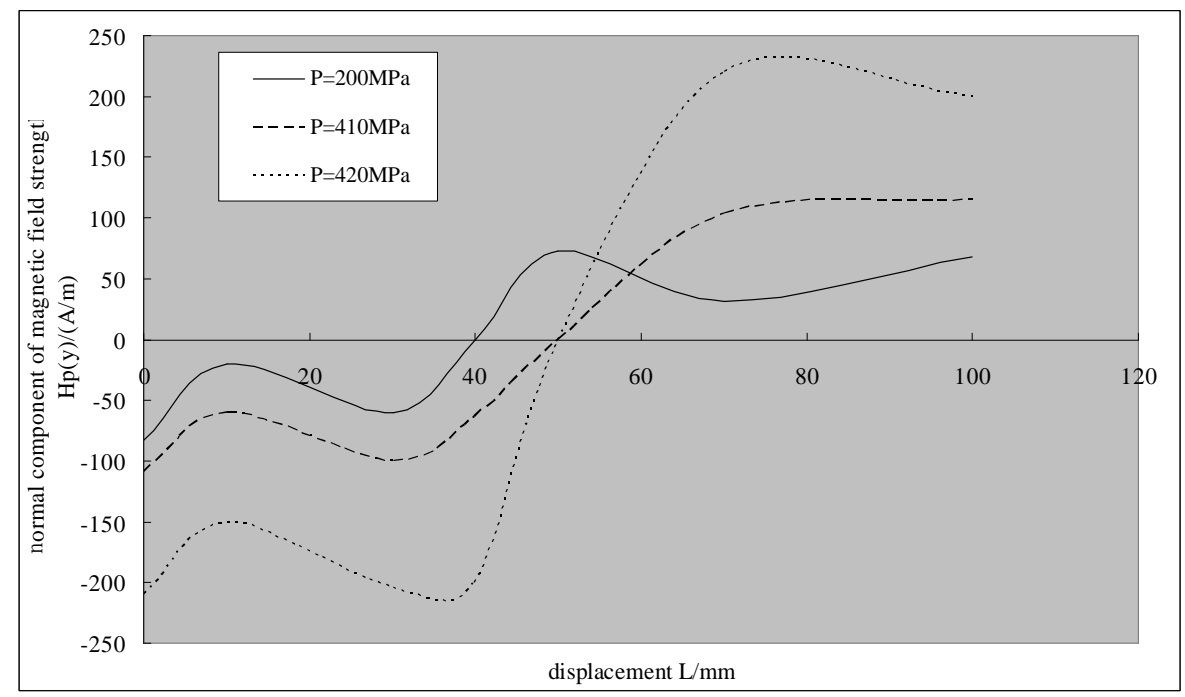

Fig. 2 MMT signals detected under tension load. 


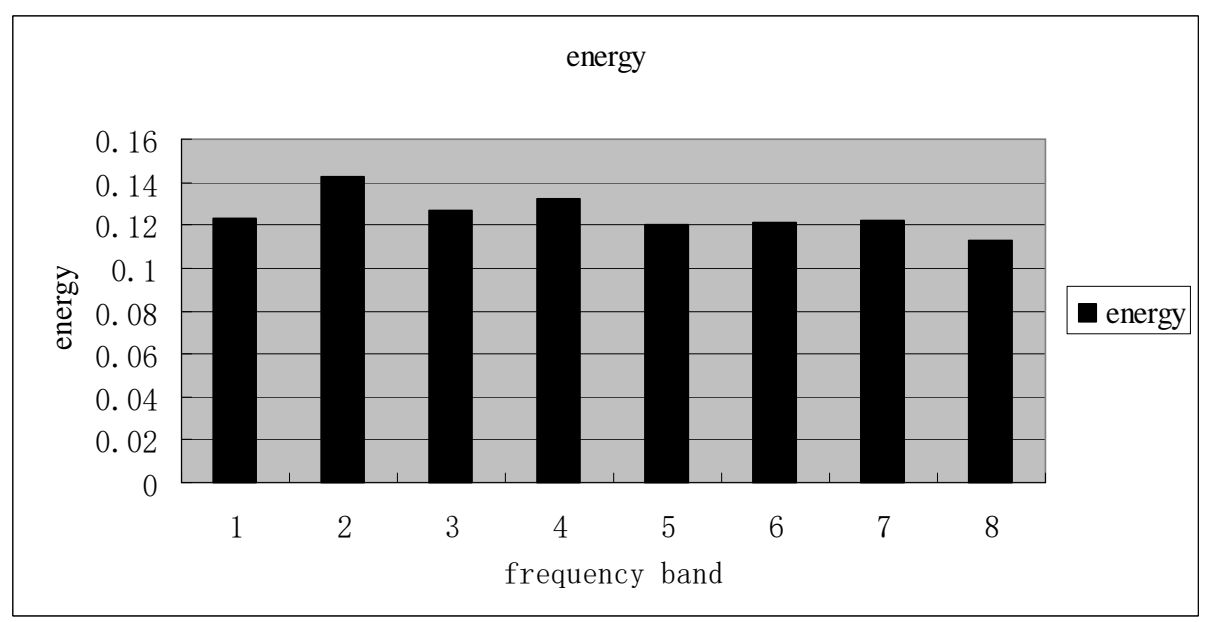

Fig. 3 wavelet packet energy spectrum, $\mathrm{P}=200 \mathrm{Mpa}$.

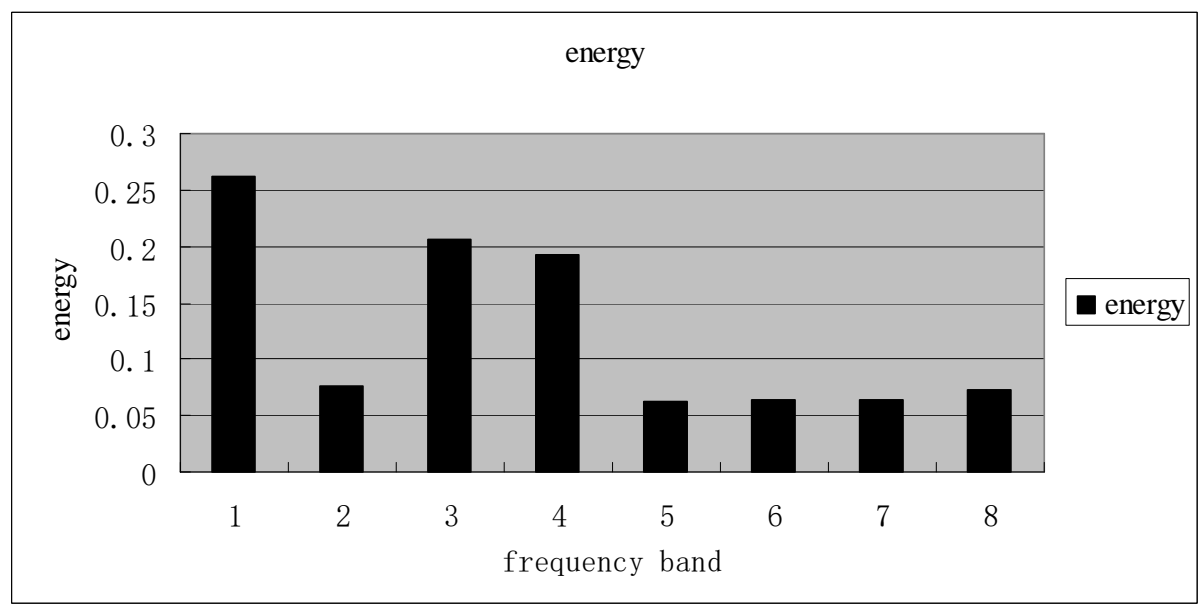

Fig. 4 wavelet packet energy spectrum, $\mathrm{P}=410 \mathrm{Mpa}$.

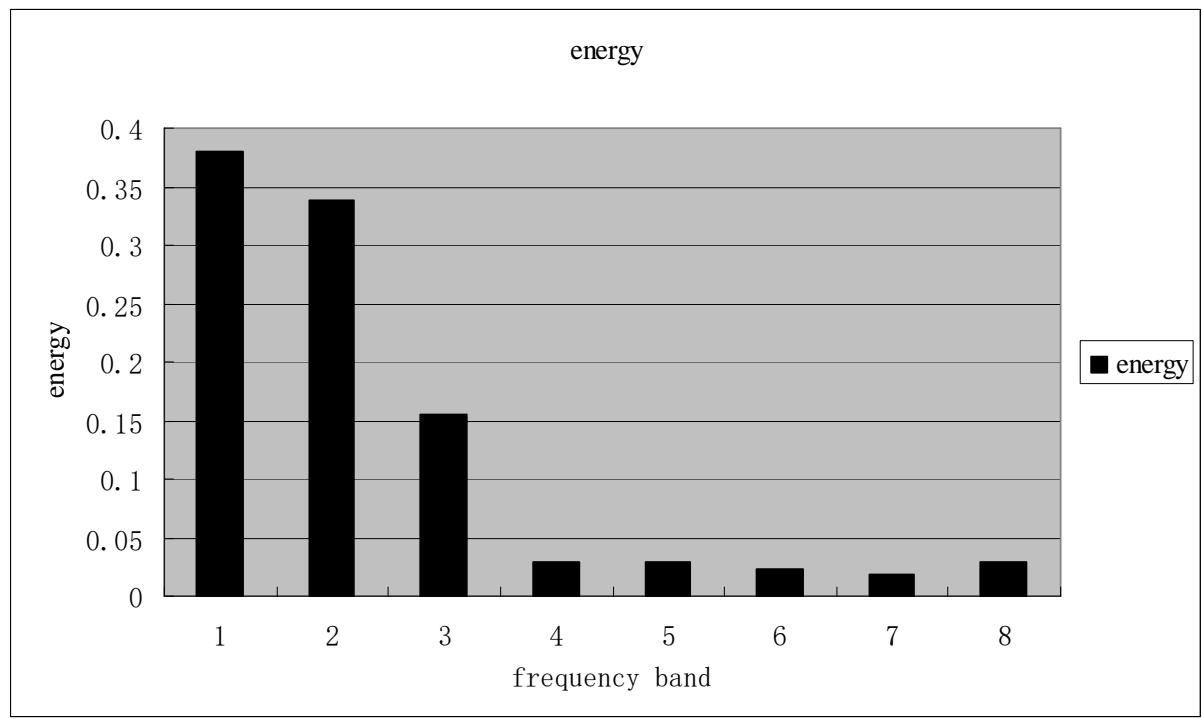

Fig. 5 wavelet packet energy spectrum, $\mathrm{P}=420 \mathrm{Mpa}$.

As can be seen from Fig. 3-5, when the tensile stress of specimen is $\mathrm{P}=200 \mathrm{MPa}$, the test specimen is in the elastic strain stage, and the degree of stress concentration is low. The wavelet packet energy spectrum distribution of the signal is relatively uniform. The wavelet packet energy of each frequency band is less than $15 \%$ of the total energy. There is no centralized distribution of frequency bands. With the increase of tensile stress, When the tensile stress of the specimen is increased to $\mathrm{P}=410 \mathrm{MPa}$, 
the maximum value of the wavelet packet energy is distributed in 1, 3 and 4 frequency bands, and the sum of 1 4 band wavelet packet energy is $73.8 \%$ of the total energy. The wavelet packet energy spectrum is mainly concentrated in the low frequency band. After the test piece, the maximum value of energy spectrum of signal wavelet packet is distributed in the 1 and 2 frequency bands, and the sum of $1 \sim 3$ band wavelet packet energy is $87.3 \%$ of the total energy. Energy spectrum distribution is extremely dispersed, and the main energy is concentrated in the 1 and 2 frequency bands of the low frequency.

It can be seen that the higher the degree of stress concentration is, the more concentrated the distribution of energy spectrum is to be. And the Energy spectrum is concentrated to the low frequency band. After the magnetic memory signal is decomposed to 3 layers by wavelet packet, the frequency band energy information can be used to characterize the stress concentration of the specimen. Thus, it can further characterize the crack. Therefore, the spectral energy of each frequency band of the magnetic memory signal forms a certain pattern in numerical value, which has a close relationship with the corresponding defects. So the wavelet packet energy spectrum of the magnetic memory signal can be extracted as the feature vector, which is used as the input vector of the intelligent identification of pipeline defects.

\section{Conclusions}

Along with the increase of tensile test piece load, the wavelet packet energy of magnetic memory signal is concentrated in the low frequency band. The greater the load, the greater the degree of concentration. The concentration of energy will reach the maximum after the test piece. The wavelet packet energy spectrum of the magnetic memory signal represents the pipeline defect. So, the wavelet packet energy spectrum of the magnetic memory signal can be extracted as the feature vector, which is used as the input vector of the intelligent identification of pipeline defects in the future.

\section{Acknowledgements}

This work was financially supported by the Chongqing Postdoctoral Science Foundation.

\section{References}

[1] A. A. Doubov, A study of metal properties using the method of magnetic memory, Metal Sci. Heat Treat. 39(9) (1997) 401-402.

[2] A. A. Doubov, The express technique of welded joints examination with use of metal magnetic memory, NDT\& E Int. 33(6) (2000) 351-362.

[3] Z. F. Liang, W. S. Li, Y. N. Wang, Zero Value Character of Metal Magnetic Memory Signal, J. Tianjin Univ. 39(7) (2006). In Chinese

[4] Z. F. Liang, Y. N. Wang, W. S. Li, General character of metal magnetic memory signal in tension experiment, J. Harbin Inst. Technol. 41(5) (2009) 99-101. In Chinese

[5] B. Duan, T. J. Sun, Z. H. Li, Signal Processing of All-digital Inverter Welder Based on Wavelet, J. Mech. Eng. 46(4) (2010) 60-63. In Chinese

[6] Y. L. Ding, A. Q. Li, Y. Deng, Parameters for identification of wavelet packet energy spectrum for structural damage alarming, J. Southeast Univ. (Natural Science Edition), 41(4) (2011) 824-825. In Chinese

[7] F. Yi, Z. X. Li, S. Yi. Design and Application of MMM/MFL Detector Based on Dual Processors, Comput. Syst. Appl. 18(9) (2009) 127-131. In Chinese

[8] S. J. Liu, Z. X. Li, S. Yi, Application of Denoising Method Based on Wavelet Packet in Metal Magnetic Memory Testing Signal Processing, Proceedings of the 2010 International Conference on 
Information Technology and Scientific Management, Tianjin, China: Scientific Research Publishing, USA, 2010:897-900. In Chinese 